\title{
Lapurdum
}

Euskal ikerketen aldizkaria | Revue d'études basques |

Revista de estudios vascos | Basque studies review

$7 \mid 2002$

Numéro VII

\section{La frontière, le territoire et le lieu. Norme et transgression dans les Pyrénées Occidentales}

\author{
Marie-Hélène Velasco-Graciet
}

\section{OpenEdition}

Journals

Édition électronique

URL : http://journals.openedition.org/lapurdum/1048

DOI : 10.4000/lapurdum.1048

ISSN : 1965-0655

\section{Éditeur}

IKER

Édition imprimée

Date de publication : 1 octobre 2002

Pagination : 347-348

ISBN : 2-86781-321-2

ISSN : $1273-3830$

Référence électronique

Marie-Hélène Velasco-Graciet, « La frontière, le territoire et le lieu. Norme et transgression dans les Pyrénées Occidentales », Lapurdum [En ligne], 7 | 2002, mis en ligne le 01 juin 2009, consulté le 10 décembre 2020. URL : http://journals.openedition.org/lapurdum/1048 ; DOI : https://doi.org/10.4000/ lapurdum.1048 
Den bezala noizpeit agian argitarat ikhusiko du... bizi denak ${ }^{2}$

Hala izan bedi.

\section{Bibliografia}

1. Dibildos, 1926, “L’Abbe Maurice Harriet et sa Soeur Mademoiselle Agathe », Gure Herria, 598-680.

2. Daranatz, 1923, "Le dictionnaire Basque Espagnol Français de l'abbé R.M. de Azkue », RIEV XIV, 457-462.

3. __ 1931, «Correspondance du Capitaine Duvoisin », RIEV, 57.

4. Knörr E., 1986, "Maurice Harriet-en hiztegiaz », ASUU, 413-417.

5. _, 1987, Para una edición critica del diccionario de Maurice Harriet. EHU (Euskal Herriko unibertsitatea) / Université du Pays-Basque, Vitoria/Gasteiz (tesi argitaragabea).

6. Lhande, 1926, Dictionnaire Basque-Français, Gabriel Beauchesne ; Paris.

\section{Marie-Hélène VELASCO-GRACIET}

\section{La frontière, le territoire et le lieu. Norme et transgression dans les Pyrénées Occidentales ${ }^{3}$.}

Depuis qu'elles existent, les frontières nationales ont intéressé les géographes. D'ailleurs, la géographie est la seule discipline scientifique à avoir consacré un de ses axes de recherches à la seule étude des frontières: la géographie des frontières. Cette géographie s'est donc construite et a connu une histoire particulière. Au fil des années de cette construction, les géographes ont constitué un corpus théorique et méthodologique La géographie des frontières connaît aujourd'hui un regain d'intérêt de la part des géographes qui se penchent à nouveau sur "l'appareillage" à mettre en place pour mieux comprendre les frontières. S'intéresser aux frontières nationales n'exclut cependant pas la possibilité d'appréhender la frontière de façon générale, qu'elle soit nationale ou non, spatiale ou non. Plus encore, traiter de frontière sous n'importe quelle forme que ce soit peut constituer une base pertinente pour une meilleure compréhension des frontières spatiales et des frontières nationales. Avant de s'intéresser aux frontières séparant les nations, l'étude de la spatialisation des frontières peut se faire pas à pas, en allant des frontières de l'individu jusqu'à celles de groupes restreints. De ce processus de recherche allant du général au particulier, une approche théorique peut être dégagée. Si l'étude des frontières spatiales ou non, de l'individu au groupe, est pertinente, c'est parce qu'elle permet de déconstruire l'objet de recherche qu'est la frontière et de le traiter comme un concept abstrait dégagé de l'imbroglio social qui se cache dans le sens commun. Ainsi, mise à nu, la frontière nationale peut être étudiée comme faisant partie d'un processus de territorialisation d'une

\footnotetext{
${ }^{2}$ Eskualdun Ona 8.zenb. 1904. urtea

3 Il s'agit d'un résumé de ma thèse soutenue en décembre 1998 à l'Université de Pau et des Pays de l'Adour.
} 
notion bien abstraite : la nation. Cette démarche permet de conceptualiser la frontière sous différents angles qui iraient de la frontière vue au prisme des sociétés qui la créent jusqu'aux frontières vues au prisme de l'intimité des sujets qui les vivent et les pratiquent au quotidien. Ce serait donc aller vers une proposition théorique pour la géographie des frontières.

Choisir comme terrain d'étude la frontière franco-espagnole, c'est s'intéresser à l'une des frontières les plus anciennes et les plus stables d'Europe Occidentale. Parce qu'elle est ancienne et stable, on la dit "froide" ou "morte". Malgré cela, il apparaît qu'elle a un intérêt scientifique pour le géographe surtout s'il porte son regard sur les différents aspects qu'elle prend. Elle est, d'une part, imposée conjointement par les Etats français et espagnol et, d'autre part, vécue dans les marges du territoire national qu'elle délimite. Pour cela, des méthodes d'observations différenciées s'imposent. La frontière franco-espagnole possède une histoire construite à force de négociations et de traités, qui lui ont progressivement donné une matérialité physique, qui s'est enrichie d'une matérialité humaine avec l'arrivée des douaniers près des frontières. Mais plus encore, par différents procédés sociaux et culturels et avec l'aide de toute une imagerie populaire, elle est entrée dans la conscience des membres de la société nationale et de ce fait, elle est devenue naturelle et comme allant de soi. Cette frontière matérielle et mise en conscience est imposée aux populations frontalières qui la côtoient quotidiennement. Au lieu de la rejeter car extérieure, les groupes frontaliers vont se l'approprier, la faire entrer dans leur vision du monde et même pluś, elle va venir conforter les pratiques et les représentations qui fondent leurs structures et leurs fonctionnements sociaux endogènes. Elle va, d'une part, renforcer les normes spatiales, sociales et culturelles du groupe ; elle fera partie du lieu-village qui est propre et singulier au groupe, elle se verra incluse dans ses récits identitaires. Et, d'autre part, Elle viendra renforcer, d'autre part, des pratiques et des pensées transgressives qui dans un lieu singulier -l'anté-lieu-, s'opèrent. L'anté-lieu et le lieu du village sont liés dans une même logique spatiale, sociale et culturelle. $\mathrm{Si}$, aujourd'hui, la frontière n'existe théoriquement plus, les populations frontalières en gardent un souvenir prégnant, le souvenir d'un temps où elles avaient trouvé un équilibre spatial, social et culturel, et où elles acceptaient de vivre dans un espace tronqué.

\title{
Mirentxu IRIGARAY
}

\author{
Écriture pour enfants, traduction et réécriture dans Bambulo de Bernardo Atxaga - \\ "ternuako penak"/ "amigos que cuentan" ("les peines de Terre-Neuve"/ "des amis qui \\ comptent/content"), Mémoire de maîtrise, dir. Mmes Geneviève CHAMPEAU et \\ Aurélie ARCOCHA-SCARCIA, soutenu le 8 octobre 2002 à l'Université Michel de \\ Montaigne, Bordeaux 3 (résumé).
}

Ce livre pour enfants commence un soir de Noël. Les personnages principaux nous sont présentés, dont Pantxi Baratxuri Buru ou Panchi Cabecita de Ajo (Pantxi Petite Tête d'Ail, en français). De même, on nous parle d'une famille composée de Maria (la mère, chargée de nettoyer la Bibliothèque de Bidebarrieta dont il est question dans Bambulo), ses trois enfants, Ariadna. Jon et Ainhoa (la petite dernière de cinq ans), et leur père, simplement désigné par "le marin de Terre-Neuve". Ce dernier, revenu d'un long voyage durant lequel il a péché la morue, raconte l'histoire d'Urkizu, un jeune marin orphelin de 